\title{
Studies of the process of moisture exchange across a membrane using irreversible thermodynamics
}

\author{
WANG LiNing \& MIN JingChun* \\ School of Aerospace, Tsinghua University, Beijing 100084, China
}

Received July 5, 2010; accepted September 26, 2010; published online May 10, 2011

\begin{abstract}
On the basis of non-equilibrium thermodynamic theory, the coupling phenomena of heat and mass transfer during the process of moisture exchange across a membrane were studied and the relevant physical and mathematical models were established. Formulae for calculating the four characteristic parameters included in the non-equilibrium thermodynamic model were derived, and the dependences of these parameters on the temperatures and concentrations on the two sides of the membrane were analyzed, providing a basis for calculating the heat and mass fluxes. The effects of temperature and concentration differences between the two sides of membrane and the membrane average temperature on the transmembrane mass and heat fluxes were investigated. The results show that for a given membrane average temperature, a larger concentration difference or a smaller temperature difference leads to a higher mass flux. For fixed concentration and temperature differences and with the mass flux predominantly caused by the concentration difference, a higher membrane average temperature yields a higher mass flux. The ratio of the heat of sorption induced by mass flow to total heat relates not only to the temperature and concentration differences between the two sides of membrane but also to the membrane average temperature and the ratio increases when the temperature difference is reduced.
\end{abstract}

transmembrane moisture exchange, heat and mass transfer, irreversible thermodynamics, characteristic parameter

Citation: Wang L N, Min J C. Studies of the process of moisture exchange across a membrane using irreversible thermodynamics. Chinese Sci Bull, 2011, 56: 1836-1843, doi: 10.1007/s11434-010-4238-5

Gas membrane separation is a new technology that has a wide range of applications, such as dew point adjustment of natural gas, energy and moisture recovery in a membrane-based total heat exchanger, and separation of organic gases. Coupled heat and mass transfer phenomena often occur in a gas separation process. For example, Wang et al. [1] claimed that the heat and mass transfer in membrane distillation is a complicated, interactive process; Phattaranawik and Jiraratananon [2,3] studied the heat and mass transfer in a direct contact membrane distillation; Alves and Coelhoso [4] analyzed the heat and mass transfer in a pervaporation process; Soowhan and Mench [5] experimentally investigated the thermo-osmosis phenomena using various membranes; and Niu and Zhang [6] discussed the impacts of various factors on the heat and mass transfer in a trans-

*Corresponding author (email: minjc @tsinghua.edu.cn) membrane moisture exchange process.

Transmembrane moisture transfer is a typical gas separation process. Studies on such processes not only have a practical value but also can provide a theoretical reference for heat and mass coupling phenomena of this kind. There are analyses of moisture transfer across membranes in the literature. For instance, $\mathrm{Hu}$ et al. [7] studied the effects of mass transfer on heat transfer; Su and Min [8] derived the moisture and heat transfer resistances through membranes; and $\mathrm{Min}$ and $\mathrm{Su}$ [9] made a further analysis of such resistances. The current work focuses on the analysis of the membrane transport process itself, using irreversible thermodynamics, which has been successfully applied to various combined transport processes.

Irreversible thermodynamics has been widely used to comprehend and quantitatively describe the coupling phenomena. Wang and Min [10] used irreversible thermody- 
namics to analyze simultaneous heat and mass transfer in a gas adsorption process at a non-equilibrium steady state. Hwang [11] pointed out that membrane transport processes were all irreversible and the description of such processes needs to follow the basic laws of irreversible thermodynamics. She and Hwang [12] used non-equilibrium thermodynamic theory to analyze the impacts of concentration, temperature and other factors on the pervaporation of inorganic solutions. Kuhn et al. [13] found that a non-equilibrium thermodynamic model could reveal the physical nature more completely than the commonly used Maxwell-Stefan model. In the current work, the transmembrane moisture exchange process model is based on irreversible thermodynamic theory. Heat and mass coupling phenomena involved in such processes are analyzed and formulae for various characteristic parameters are derived. Using these as a base, the relations between the transmembrane fluxes and the temperature and concentration gradients are studied and the relation between the ratio of the heat of sorption induced by mass transfer to total heat and various parameters is discussed.

\section{Theoretical models}

\subsection{Physical model}

Consider the process of water vapor transfer through a nonporous membrane, as shown in Figure 1, where the water vapor concentrations on the two sides of membrane are $C_{1}$ and $C_{2}$, and the temperatures are $T_{1}$ and $T_{2}$, respectively, with $C_{1}>C_{2}$ and $T_{1}>T_{2}$. According to the solution-diffusion theory, water vapor will transfer from the side with higher humidity to that with lower humidity due to the concentration and temperature gradients. To highlight the process of moisture transfer across membrane itself, the convective heat and mass transfer resistances on the two sides of membrane are neglected, that is, the concentration and temperature in the space on each side of membrane are considered to be constant (i.e., there are neither concentration nor temperature boundary layers).

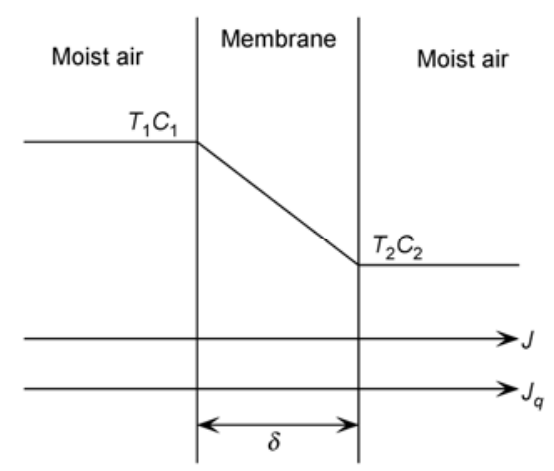

Figure 1 Diagram of heat and mass transfer through membrane.

\subsection{Non-equilibrium thermodynamic model}

For a combined heat and mass transfer process, if the driving forces are the concentration and temperature differences, the entropy production rate can be written as [14]

$$
\sigma=J_{q} \frac{\nabla T}{T^{2}}+J \frac{\nabla C}{T} .
$$

The linear phenomenological equations for the corresponding heat and mass fluxes are

$$
\left\{\begin{array}{l}
J_{q}=L_{q q} \frac{\nabla T}{T^{2}}+L_{q w} \frac{\nabla C}{T}, \\
J=L_{w q} \frac{\nabla T}{T^{2}}+L_{w w} \frac{\nabla C}{T},
\end{array}\right.
$$

where $L$ is a phenomenological coefficient that reflects the phenomenological relations between the thermodynamic flux and force. For convenience in analysis and comprehension, four characteristic parameters are introduced to express the phenomenological coefficients [15]:

Thermal conductivity: $\lambda=L_{q q} / T^{2}$;

Molar-driven thermal conductivity: $\lambda^{\prime}=L_{q w} / T$;

Thermally driven molar conductivity: $\lambda_{m}^{\prime}=L_{q w} / T^{2}$;

Molar conductivity: $\lambda_{m}=L_{w w} / T$.

Substituting the above four characteristic parameters into eq. (2) yields

$$
\left\{\begin{array}{l}
J_{q}=\lambda \nabla T+\lambda^{\prime} \nabla C, \\
J=\lambda_{m}^{\prime} \nabla T+\lambda_{m} \nabla C .
\end{array}\right.
$$

It can be determined from the above expressions that there are two advantages arising from the non-equilibrium thermodynamic model. First, the model can directly take into account the interactive effects caused by two or more irreversible phenomena that interact, e.g., the mutual coupling of the heat and mass transfer involved in the present model, in which the coupling relation between the irreversible phenomena is represented by the phenomenological coefficients, which are the molar-driven thermal conductivity and thermally driven molar conductivity. Second, the relationships between the thermodynamic forces and fluxes are simple and straightforward and can be observed directly from the equations, with the thermodynamic forces and fluxes linked together by the phenomenological coefficients. In this paper, the thermodynamic forces and fluxes are related by the thermal conductivity, molar-driven thermal conductivity, molar conductivity, and thermally driven molar conductivity. The magnitudes of the characteristic parameters reflect the contribution rates of various driving forces for the thermodynamic fluxes, e.g., for the mass transfer in this work, a large molar conductivity implies a strong effect of the concentration difference.

\subsection{Mass transfer mechanism}

The process of gas transfer across a membrane is generally 
described using the solution-diffusion model, which considers that the gas first adsorbs at the membrane surface on one side, then diffuses through the membrane, and eventually desorbs from the membrane surface on the other side. For the transmembrane moisture transfer process considered here, the concentrations on the two sides of the membrane are not high, so Henry's law can be used to describe the gas adsorption and desorption behaviors at the two membrane surfaces, i.e.

$$
\theta=S \times C,
$$

where $\theta$ is the adsorptive capacity of the membrane surface, $S$ is the solubility coefficient, and $C$ is the gas concentration at the membrane surface.

Fick's law can be used to describe the process of moisture transfer inside the membrane as follows:

$$
J=\frac{D}{\delta}\left(\theta_{1}-\theta_{2}\right),
$$

where $D$ is the diffusion coefficient of water vapor in the membrane $\left(\mathrm{kg} \mathrm{m}^{-1} \mathrm{~s}^{-1}\right)$ and $\delta$ is the membrane thickness.

In addition, the formulae for calculating the solubility and diffusion coefficient can be written as [16]

$$
S=S_{0} \mathrm{e}^{-\Delta H_{s} / R T}, D=D_{0} \mathrm{e}^{-E_{d} / R T},
$$

where $D_{0}$ is the diffusion constant and $S_{0}$ is the solubility constant, both of which are independent of temperature, $E_{d}$ is the energy of diffusion activation, and $\Delta H_{s}$ is the heat of solution.

Substituting eqs. (4) and (6) into eq. (5) gives

$$
J=\frac{D_{0} S_{0} \mathrm{e}^{-E_{d} / R T_{\text {ave }}}}{\delta}\left(\mathrm{e}^{-\Delta H_{s} / R T_{1}} \times C_{1}-\mathrm{e}^{-\Delta H_{s} / R T_{2}} \times C_{2}\right) .
$$

The above equation describes the mass flux across membrane, in which the diffusion coefficient is related to the temperature of membrane and is determined by the average temperature of membrane, $T_{\text {ave }}$, whereas the adsorption and desorption occur at the membrane surfaces so are determined by the absolute temperatures of the membrane surfaces.

In order to suit the form of the irreversible thermodynamic model, the above equation can be further transformed into:

$$
\begin{gathered}
J=\frac{D_{0} S_{0} \mathrm{e}^{-E_{d} / R T_{\text {ave }}}}{\delta} \\
\times\left(C_{2}\left(\mathrm{e}^{-\Delta H_{s} / R T_{1}}-\mathrm{e}^{-\Delta H_{s} / R T_{2}}\right) \frac{\Delta T}{\Delta T}+\left(C_{1}-C_{2}\right) e^{-\Delta H_{s} / R T_{1}}\right), \\
\text { or } \quad J=\frac{A B\left(\mathrm{e}^{X}-\mathrm{e}^{Y}\right)}{\Delta T} \nabla T+A \mathrm{e}^{X} \nabla C,
\end{gathered}
$$

where

$$
A=D_{0} S_{0} \mathrm{e}^{-E_{d} / R T_{\text {ave }}}, \quad B=C_{\text {ave }}-\Delta C / 2
$$

$$
\begin{gathered}
X=-\Delta H_{s} /\left(R \times\left(T_{\text {ave }}+\Delta T / 2\right)\right), \\
Y=-\Delta H_{s} /\left(R \times\left(T_{\text {ave }}-\Delta T / 2\right)\right),
\end{gathered}
$$

$\Delta C=C_{1}-C_{2}$ and $\Delta T=T_{1}-T_{2}$ express the concentration and temperature differences, respectively, while $T_{\mathrm{ave}}=\left(T_{1}+T_{2}\right) / 2$ and $C_{\mathrm{ave}}=\left(C_{1}+C_{2}\right) / 2$ express the membrane average temperature and concentration, respectively. It can be seen from eq. (8) that $A B\left(\mathrm{e}^{X}-\mathrm{e}^{Y}\right) / \Delta T=\lambda_{m}{ }^{\prime}$, which suggests that the thermally driven molar conductivity $\lambda_{m}^{\prime}$ is related not only to the membrane average temperature and concentration but also to the temperature and concentration differences between the two sides of membrane. That is, the characteristic parameter corresponding to the temperature difference relates not only to the temperatures but also the concentrations on the two sides of membrane. Also, $A \mathrm{e}^{X}=\lambda_{m}$, which indicates that the molar conductivity $\lambda_{m}$ is related to the membrane average temperature and the temperature difference between the two sides of membrane. That is, the concentration difference corresponding to the characteristic parameter is determined by the temperatures on the two sides of membrane. In summary, the corresponding relationships between the characteristic parameters and driving forces reflect the combination of heat and mass transfer.

\subsection{Heat transfer mechanism}

The heat transferred through the membrane includes the heat driven by the temperature gradient and that carried by the mass flow, where the conduction heat flux caused by the temperature gradient is $j_{q d}=\lambda_{e} \nabla T$ and the heat flux caused by the mass flux is $J_{q c}=J \Delta h$. The total heat flux $J_{q}$, therefore, can be expressed as

$$
J_{q}=J_{q d}+J_{q c}=\lambda_{e} \nabla T+J \Delta h,
$$

where $\lambda_{e}$ is the effective thermal conductivity of membrane and $J$ is the mass flux across membrane. Substitution of eq. (8) into eq. (10) yields the relationship between the heat flux and the temperature and concentration differences:

$$
J_{q}=\left(\lambda_{e}+\lambda_{m}^{\prime} C_{p} \Delta T\right) \nabla T+\lambda_{m} C_{p} \Delta T \nabla C .
$$

Furthermore, comparison of eq. (11) with eq. (13) can offer the thermal conductivity and molar-driven thermal conductivity: $\lambda=\lambda_{e}+\lambda_{m}^{\prime} C_{p} \Delta T$ and $\lambda^{\prime}=\lambda_{m} C_{p} \Delta T$, respectively. These two characteristic parameters both depend on the temperatures and concentrations on the two sides of membrane, implying that the heat flux is also determined by the concentrations and temperatures on the two sides of membrane.

For the process of water vapor transfer through membrane, the heat transferred inside the membrane should be equal to that outside the membrane, which includes two parts: one is the convective heat outside the membrane, namely the sensible heat $q_{1}$, and the other is the heat of sorption due to the water vapor adsorption or desorption at 
the membrane surface, namely the latent heat $q_{2}$, which can be represented by

$$
q_{2}=J L
$$

$L$ is the heat of sorption. So, the total heat flux is

$$
J_{q}=q_{1}+q_{2}
$$

If the ratio of latent to total heat is expressed as

$$
k=q_{2} / J_{q},
$$

it can be seen from eqs. (12) and (13) that $k$ represents the fraction of the heat of sorption caused by the mass flow relative to the total heat.

\section{Results and discussion}

Using the heat and mass transfer of humid air across membrane as an example, the coupling phenomena of membrane transport were studied using irreversible thermodynamic theory, the value of each characteristic parameter was calculated, and the effects of the temperature and concentration differences between the two sides of membrane and the membrane average temperature on the mass and heat transfer were analyzed. The values taken for some key parameters are as follows: $\lambda_{e}=0.1 \mathrm{~W} \mathrm{~m}^{-1} \mathrm{~K}^{-1}, \delta / D_{0} S_{0}=0.002 \mathrm{~kg}$ $\mathrm{m}^{-1} \mathrm{~s}^{-1}, E_{\mathrm{d}}=50 \mathrm{~kJ} \mathrm{~mol}^{-1}$, and $\Delta H_{s}=-25 \mathrm{~kJ} \mathrm{~mol}^{-1}$. Because the relative humidity needs to satisfy the condition $\phi \leqslant 1$, and the relation between the relative humidity and concentration is $\phi / C=\mathrm{e}^{5294 / \mathrm{T}} / \rho_{\mathrm{a}} 10^{6}$, the concentrations on the two sides of membrane must obey the relation $C \leqslant 10^{6} \rho_{\mathrm{a}} / \mathrm{e}^{5294 / T}$, where $\rho_{\mathrm{a}}$ is the air density, whose value is taken to be $\rho_{\mathrm{a}}=1.27 \mathrm{~kg}$ $\mathrm{m}^{-3}$.

\subsection{Characteristic parameters}

The phenomenological coefficients or characteristic parameters involved in the non-equilibrium thermodynamic model are generally assigned empirical values obtained through experiments under certain conditions. In the present work, however, specific formulae for calculating the characteristic parameters applying to the near equilibrium states at various conditions were obtained through theoretical analyses. The formulae for the four characteristic parameters are summarized as follows.

Thermally driven molar conductivity:

$$
\lambda_{m}^{\prime}=\frac{A B\left(\mathrm{e}^{X}-\mathrm{e}^{Y}\right)}{\Delta T},
$$

molar conductivity: $\lambda_{m}=A \mathrm{e}^{X}$.

Thermal conductivity:

$$
\lambda=\lambda_{e}+\lambda_{m}^{\prime} C_{p} \Delta T=\lambda_{e}+A B\left(\mathrm{e}^{X}-\mathrm{e}^{Y}\right) C_{p} .
$$

Molar-driven thermal conductivity:

$$
\lambda^{\prime}=\lambda_{m} C_{p} \Delta T=A \mathrm{e}^{X} C_{p} \Delta T .
$$

The above four expressions indicate that the characteristic parameters included in this model are all related to the membrane operating conditions. The thermally driven molar conductivity and the thermal conductivity are related to not only the temperatures but also the concentrations on the two sides of the membrane, whereas the molar conductivity and the molar-driven thermal conductivity are related only to the temperatures on the two sides of the membrane. As described by eq. (4), the temperature difference affects the mass flux by altering the solubility coefficient and consequently the adsorptive capacities at the two surfaces of the membrane. Noting that adsorption is the product of the solubility coefficient and concentration, the characteristic parameter corresponding to the mass flux induced by the temperature gradient needs to include information on the concentrations at the membrane surfaces. The characteristic parameters corresponding to the concentration difference must also include information on the solubility, which is a function of temperature, so they are also functions of temperature. However, it should be pointed out that because the mass and heat fluxes are functions of both temperature and concentration, the product of each characteristic parameter and its corresponding force must also be a function of temperature and concentration. The specific expressions for the characteristic parameters actually reflect the mutual coupling of heat and mass transfer.

Next, analyses are made on the variations of the characteristic parameters with operating conditions. The relations between the concentrations and characteristic parameters are relatively simple, i.e., the concentration level and concentration difference have linear relationships with the thermally driven molar conductivity and the thermal conductivity but are independent of the molar conductivity and the molar-driven thermal conductivity. Therefore, emphasis will be placed on the analyses of the characteristic parameters at different temperatures, for which the average concentration and concentration difference are set to be $C_{\text {ave }}=$ $0.01 \mathrm{~kg} \mathrm{~m}^{-3}$ and $\Delta C=0.01 \mathrm{~kg} \mathrm{~m}^{3}$, respectively.

Figure 2(a) and (b) show the variations of the two mass flux relating characteristic parameters, namely the thermally driven molar conductivity and the molar conductivity, with the membrane average temperature under various temperature differences. From Figure 2(a), the thermally driven molar conductivity is negative, implying that the temperature difference causes a mass flow in the direction opposite to the temperature gradient, while the molar conductivity is positive, meaning that the concentration difference induces a mass flow in the same direction as the concentration gradient. Also, the absolute value of the thermally driven molar conductivity increases with increasing membrane average temperature, because a higher membrane average temperature yields a larger diffusion coefficient, which enables the water vapor to transfer more easily through the membrane. 

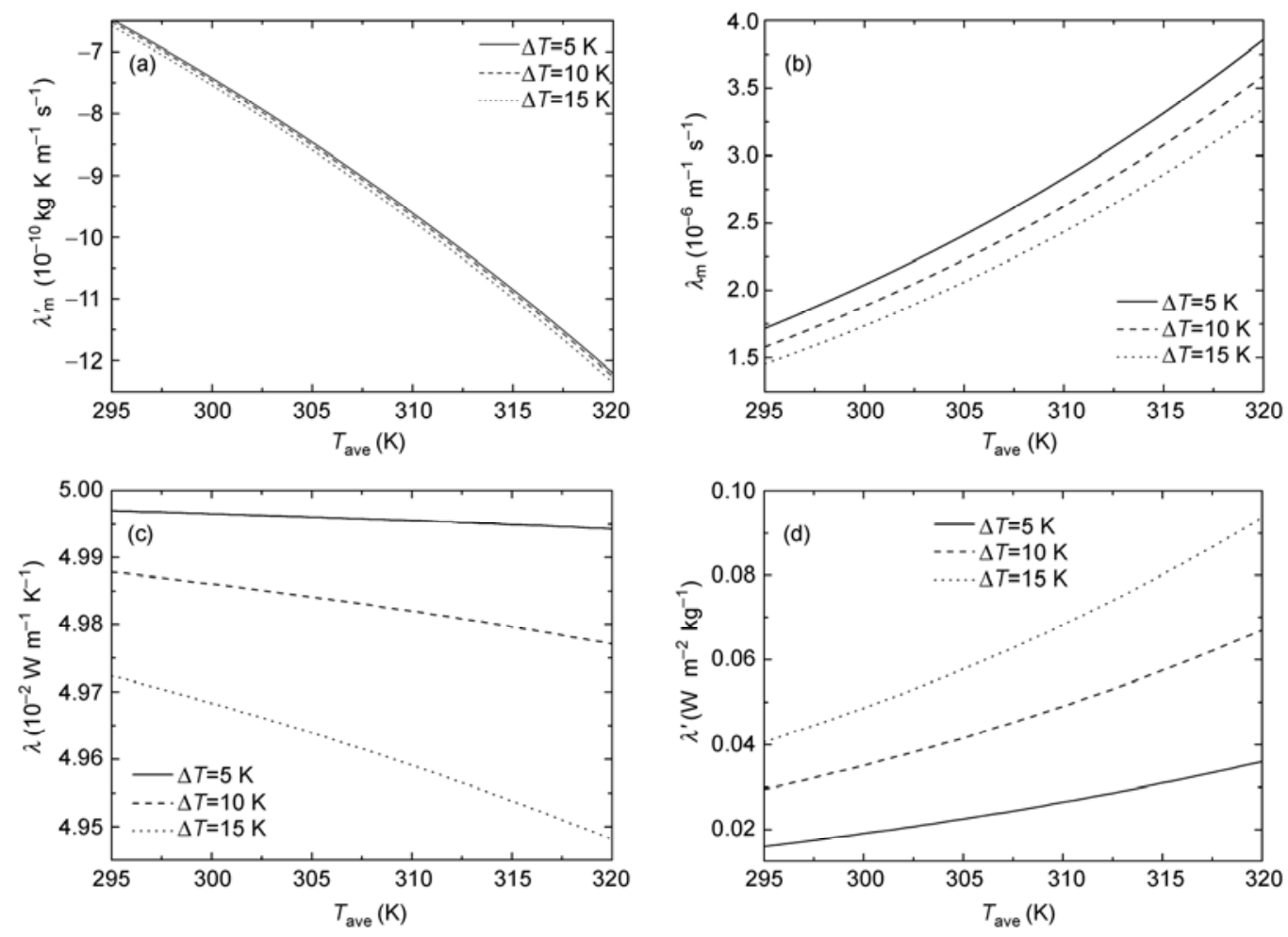

Figure 2 Variations of the four characteristic parameters with the average temperature and temperature difference. (a) Thermally driven molar conductivity; (b) molar conductivity; (c) thermal conductivity; (d) molar-driven thermal conductivity.

Figure 2(b) shows that the molar conductivity increases with increasing membrane average temperature, the reason for which is the same as that for the trend with thermally driven molar conductivity. The product of the thermally driven molar conductivity with the temperature difference, which is the mass flux caused by the temperature difference, is relatively small compared with the product of the molar conductivity and the concentration difference, which is the mass flux caused by the concentration difference. Hence, the mass flux caused by the concentration difference predominates in the total mass flux, but if the concentration difference is small, the mass flux caused by the temperature difference may also play an important role.

Figure 2(c) and 2(d) show the variations of the two heat flux relating characteristic parameters, namely the molardriven thermal conductivity $\lambda^{\prime}$ and the thermal conductivity $\lambda$, with the membrane average temperature under various temperature differences. The thermal conductivity shown in Figure 2(c) changes very slightly with the temperature difference and average temperature, with a deviation from the effective thermal conductivity $\lambda_{e}$ less than $0.6 \%$, so it is reasonable to consider that the thermal conductivity is equal to the effective thermal conductivity. The molar-driven thermal conductivity $\lambda^{\prime}$ shown in Figure 2(d) increases with increasing membrane average temperature, with a larger temperature difference giving a greater molar-driven thermal conductivity. This is because the heat flux component corresponding to $\lambda^{\prime}$ is the enthalpy carried by the mass flux component corresponding to the molar conductivity $\lambda_{m}$. Since $\lambda_{m}$ increases with the membrane average temperature, $\lambda^{\prime}$ also increases with the membrane average temperature. In addition, a larger temperature difference causes a greater value of enthalpy carried by the mass flow, which means that a greater value of enthalpy is carried by the mass flow driven by the identical concentration difference, leading to a larger molar-driven thermal conductivity, $\lambda^{\prime}$. Also, in this analysis, the thermal conductivity is almost constant at $0.05 \mathrm{~W} \mathrm{~m}^{-1} \mathrm{~K}^{-1}$, the molar-driven thermal conductivities range from 0.01 to $0.1 \mathrm{~W} \mathrm{~m}^{2} \mathrm{~kg}^{-1}$, and the temperature and concentrations differences range from 0 to $20 \mathrm{~K}$ and 0 to $0.015 \mathrm{k} \mathrm{gm}^{-3}$, respectively. The heat flux caused by the concentration difference is therefore negligible compared with the heat flux caused by the temperature difference.

\subsection{Mass flux}

Figure 3 shows the variations in mass flux with temperature difference at various average temperatures for a concentration difference $\Delta C=0.01 \mathrm{~kg} / \mathrm{m}^{3}$ and an average concentration $C_{\text {ave }}=0.015 \mathrm{~kg} / \mathrm{m}^{3}$. It should be noted that the mass flux and temperature difference is not in a simple linear relation, as can be seen from eq. (8). As the temperature difference increases, the mass flux decreases, which means 


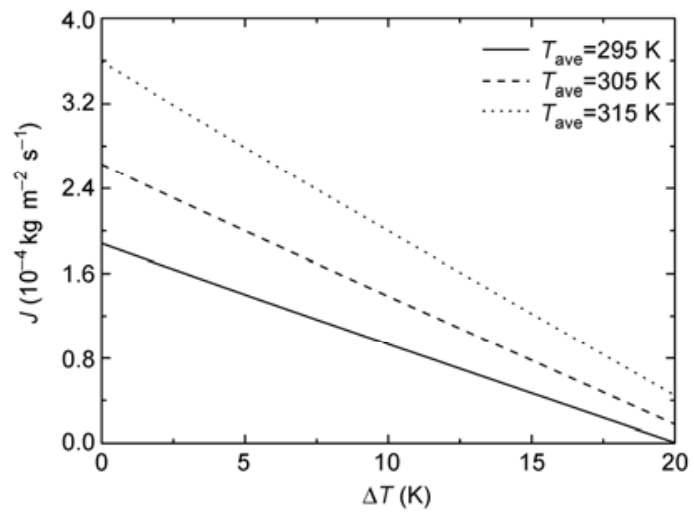

Figure 3 Variations of mass flux with temperature difference.

that the temperature difference as one of the driving forces affects the mass flux. The reason for the decrease in mass flux is that the thermally driven molar conductivity is negative, so a positive temperature difference will lead to a negative mass flux. The physical situation is that for a given average temperature, an increased temperature difference means an increased temperature $T_{l}$ and a reduced temperature $T_{2}$. According to the dependence of adsorptive capacity on temperature, the adsorptive capacity decreases at the membrane surface with high temperature but increases at the membrane surface with lower temperature, i.e., $\theta_{1}$ decreases while $\theta_{2}$ increases, as a result, the total mass flux decreases. Furthermore, it can be seen from Figure 3 that as the temperature difference decreases, the mass flux through the membrane with a high average temperature decreases more rapidly than that with a lower average temperature. The reason is that a higher average temperature yields a larger thermally driven molar conductivity $\lambda_{\mathrm{m}}^{\prime}$, so for a given temperature difference, the mass flux decreases more rapidly.

Figure 4 shows variations in mass flux with concentration difference for different temperature differences and average temperatures for $C_{\text {ave }}=0.01 \mathrm{~kg} / \mathrm{m}^{3}$. It can be seen that the concentration difference is the other driving force in the non-equilibrium thermodynamic model and that the mass flux increases as this force increases. The reason for this behavior is that the molar conductivity is positive and therefore the mass flux caused by the concentration difference is positive, which is consistent with Fick's law. When the temperature difference is constant and the concentration difference is small, a higher average temperature yields a smaller mass flux. When the concentration difference is larger, however, a higher average temperature tends to give a larger mass flux. This is because, on the one hand, the molar conductivity $\lambda_{m}$ increases with increasing average temperature, so for a given concentration difference, a higher average temperature offers a larger mass flux component caused by the concentration difference. On the other hand, the thermally driven molar conductivity $\lambda_{m}^{\prime}$ decreases with increasing average temperature, so the mass flux com- ponent caused by the temperature difference decreases with increasing average temperature. Thus, when the concentration difference is small but the temperature difference is relatively large, the mass flux caused by the temperature difference constitutes a large portion of the total mass flux and therefore a higher average temperature leads to a smaller mass flux. When the concentration difference is larger, however, the mass flux caused by the concentration difference constitutes a larger portion of the total mass flux, so a higher average temperature leads to a larger mass flux. In addition, for a given average temperature, a larger temperature difference yields a smaller mass flux because the temperature difference causes a negative mass flux component.

In summary, it can be concluded from the above analysis that the concentration and temperature differences are the driving forces for the membrane transport phenomena examined in this work and that the average temperature is closely related to the phenomenological or characteristic parameters. In practice, for moisture exchange process across a membrane, the temperature difference between the two sides of the membrane is small, so the concentration difference is the predominant driving force for the transmembrane moisture transfer.

\subsection{The ratio of heat of sorption to total heat}

In order to study the effects of the heat of sorption caused by the mass transfer on the total heat flux, analyses are made below on the relationship between the heat of sorption and total heat under different driving forces and with different characteristic parameters. Figure 5 shows the variations in the sorption to total heat ratio, $k$, with temperature difference for various average temperatures for $C_{\text {ave }}=0.015$ $\mathrm{kg} \mathrm{m}^{-3}$ and $\Delta C=0.01 \mathrm{~kg} \mathrm{~m}^{-3}$. When the temperature difference is increased, $k$ decreases. This is because the total heat flux depends mainly on the temperature difference, while the heat of sorption caused by the mass transfer is determined by both the temperature difference and concentration difference. It can be concluded from the above that as the

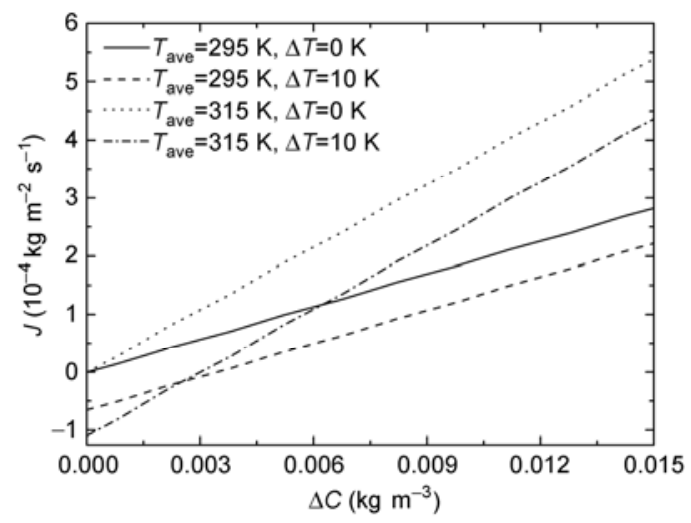

Figure 4 Variations of mass flux with concentration difference. 


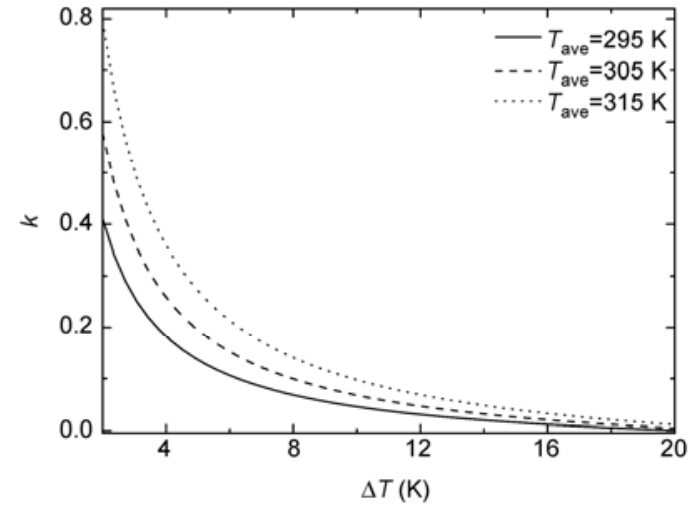

Figure 5 Variations of sorption to total heat ratio with temperature difference at various average temperatures.

temperature difference increases, the mass flux $J$ decreases but the total heat flux $J_{q}$ increases and therefore the sorption to total heat ratio decreases. Additionally, over a certain range of temperature differences, a higher average temperature yields a larger $k$. This is because for a given temperature difference, a higher average temperature corresponds to a larger molar conductivity, which increases the mass flux if the concentration difference is fixed. The heat of sorption caused by the mass flow therefore increases, leading to an increased $k$.

Figure 6 shows variations in the ratio of the heat of sorption to total heat with temperature difference for various concentration differences for $T_{\mathrm{ave}}=295.15 \mathrm{~K}$ and $C_{\mathrm{ave}}=0.01$ $\mathrm{kgm}^{-3}$. The figure shows that $k$ increases as the concentration difference increases. The reason is that a greater concentration difference yields a larger mass flux, leading to a greater ratio of the heat of sorption caused by the mass flux to the total heat flux, if the temperature difference is fixed.

\section{Conclusions}

(1) Using the irreversible thermodynamic approach, a theo-

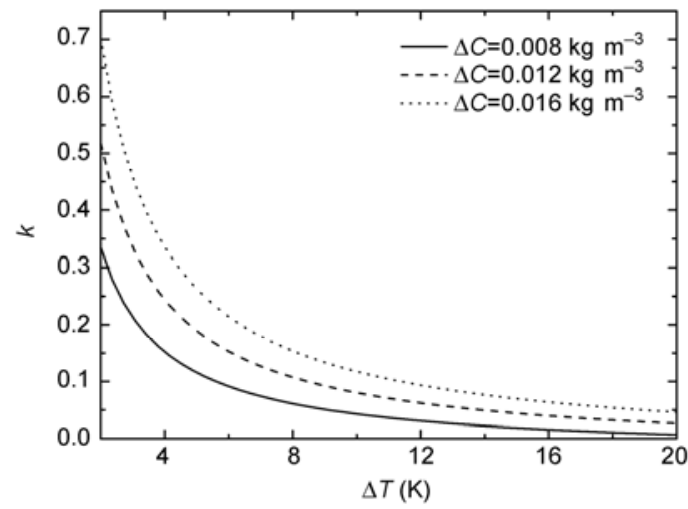

Figure 6 Variations of sorption to total heat ratio with temperature difference for various concentration differences. retical model was developed to describe the coupled heat and mass transfer in the process of moisture exchange across a membrane. The model directly relates the concentration and temperature differences, providing convenient analysis.

(2) Using the above model as a base, formulae for calculating the various characteristic parameters included in the non-equilibrium thermodynamic model were derived, and the variations of these parameters with the temperatures and concentrations at the two sides of membrane were analyzed. The results suggest that the thermally driven molar conductivity and the thermal conductivity relate not only to the temperatures but also to the concentrations at the two sides of the membrane. The molar conductivity and the molar-driven thermal conductivity, however, relate only to the temperatures at the two sides of the membrane. The relationships between the characteristic parameters and special operating conditions reflect the coupling of heat and mass transfer.

(3) The mass flux relates not only to the concentration difference but also to the membrane average temperature and the temperature difference between the two sides of membrane. For a given average temperature, a larger concentration difference or a smaller temperature difference yields a greater mass flux. For fixed concentration and temperature differences and with the mass flux component caused by the concentration difference predominating over the total mass flux, a higher average temperature gives a larger mass flux, because the diffusion coefficient increases with increasing temperature.

(4) The ratio of the heat of sorption caused by the mass transfer to the total heat is related to the temperature difference, concentration difference and average temperature. The smaller the temperature difference, the larger the portion of the heat of sorption relative to the total heat. The reason is that when the temperature difference is small, the total heat flux is also small but the heat of sorption caused by the mass flow remains large.

This work was supported by the National Natural Science Foundation of China (50576040)

1 Wang Z S, Gu Z L, Feng S Y, et al. Applications of membrane distillation technology in energy transformation process-basis and prospect. Chinese Sci Bull, 2009, 54: 2766-2780

2 Phattaranawik J, Jiraratananon R, Fanc A G. Heat transport and membrane distillation coefficient in direct contact membrane distillation. J Mem Sci, 2003, 212: 177-193

3 Phattaranawik J, Jiraratananon R. Direct contact membrane distillation: Effect of mass transfer on heat transfer. J Mem Sci, 2001, 188: 137-143

4 Alves V D, Coelhoso I M. Study of mass and heat transfer in the osmotic evaporation process using hollow fiber membrane contactors. J Mem Sci, 2007, 289: 249-257

5 Kim S, Mench M M. Investigation of temperature-driven water transport in polymer electrolyte fuel cell: Thermo-osmosis in membranes. J Mem Sci, 2009, 328: 113-120 
6 Niu J L, Zhang L Z. Membrane based enthalpy exchanger: Material considerations and clarification of moisture resistance. J Mem Sci, 2001, 189: 179-191

7 Hu T, Min J C, Song Y Z. Analysis of the effects of mass transfer in the progress of moisture exchanger across a membrane. Chinese Sci Bull, 2010, 55: 1221-1225

8 Su M, Min J C. Analysis of coupled heat and mass transfer in membrane process (in Chinese). J Chem Ind Engineer, 2008, 59: 825-829

9 Min J C, Su M. Performance analysis of a membrane-based enthalpy exchanger: Effects of the membrane properties on the exchanger performance. J Mem Sci, 2010, 348: 376-382

10 Wang L N, Min J C. Thermodynamic analysis of adsorption process at a non-equilibrium steady state. Chinese Sci Bull, 2010, 55: 3612-3618

11 Hwang S T. Nonequilibrium thermodynamics of membrane transport.
AIChE J, 2004, 50: 862-870

12 She M, Hwang S T. Effects of concentration, temperature, and coupling on pervaporation of dilute flavor organics. J Mem Sci, 2006, 271: 16-28

13 Kuhn J, Stemmer R, Kapteijn F, et al. A non-equilibrium thermodynamics approach to model mass and heat transport for water pervaporation through a zeolite membrane. J Mem Sci, 2009, 330: 388-398

14 Zeng D L. Project Non-balanced Thermodynamics (in Chinese). Beijing: Scientific Publishing House, 1991

15 Yang D H. The Principles and Engineering Applications of Irreversible Thermodynamics (in Chinese). Beijing: Science Press, 1989

16 Sun Y M, Wu C H, Lin A. Sorption and permeation properties of water and ethanol vapors in poly[bis(trifluoro-ethoxy)phosphazene] (PEFEP) membranes. J Polym Res, 1999, 6: 91-98

Open Access This article is distributed under the terms of the Creative Commons Attribution License which permits any use, distribution, and reproduction in any medium, provided the original author(s) and source are credited. 